\title{
Functional annotations of diabetes nephropathy susceptibility loci through analysis of genome-wide renal gene expression in rat models of diabetes mellitus
}

\author{
Yaomin $\mathrm{Hu}^{\dagger 1}$, Pamela J Kaisaki ${ }^{\dagger 1}$, Karène Argoud ${ }^{\dagger 1}$, Steven P Wilder ${ }^{1}$, \\ Karin J Wallace ${ }^{1}$, Peng Y Woon ${ }^{1}$, Christine Blancher ${ }^{1}$, Lise Tarnow ${ }^{2}$, Per- \\ Henrik Groop ${ }^{3}$, Samy Hadjadj4 ${ }^{4}$ Michel Marre ${ }^{5}$, Hans-Henrik Parving6, \\ Martin Farrall ${ }^{1}$, Roger D Cox ${ }^{7}$, Mark Lathrop ${ }^{8}$, Nathalie Vionnet ${ }^{9}$, Marie- \\ Thérèse Bihoreau ${ }^{1}$ and Dominique Gauguier*1,10
}

\begin{abstract}
Address: ${ }^{1 T h e}$ Wellcome Trust Centre for Human Genetics, University of Oxford, Roosevelt Drive, Oxford OX3 7BN, UK, ${ }^{2}$ Steno Diabetes Center, Copenhagen, Denmark, ${ }^{3}$ Department of Medicine, Division of Nephrology, Helsinki University Central Hospital and Folkhälsan Institute of Genetics, Helsinki, Finland, ${ }^{4}$ CHU Poitiers, University Hospital, Endocrinology and INSERM, ERM 324, Poitiers, France, ${ }^{5}$ Department of Diabetology, Bichat Hospital and INSERM, U695, Xavier Bichat University of Medicine, Paris, France, ${ }^{\circ}$ Department of Medical Endocrinology, Rigshospitalet, University Hospital of Copenhagen, Copenhagen, Denmark, ${ }^{7}$ MRC Mammalian Genome Unit, Harwell OX11 0RD, UK, ${ }^{8}$ National Genotyping Centre, Evry, France, 9 INSERM, UMR S 525, Université Pierre et Marie Curie-Paris 6, Paris, France and ${ }^{10}$ INSERM, U872, Centre de Recherche des Cordeliers, Paris, France

Email: Yaomin Hu - amin1031@hotmail.com; Pamela J Kaisaki - pkaisaki@well.ox.ac.uk; Karène Argoud - karene@well.ox.ac.uk; Steven P Wilder - swilder@ebi.ac.uk; Karin J Wallace - karin.wallace@gmail.com; Peng Y Woon - pengwoon@gmail.com; Christine Blancher - christin@well.ox.ac.uk; Lise Tarnow - ltar@steno.dk; Per-Henrik Groop - per-henrik.groop@helsinki.fi; Samy Hadjadj - s.hadjadj@chu-poitiers.fr; Michel Marre - michel.marre@bch.aphp.fr; Hans-Henrik Parving - HHParving@dadlnet.dk; Martin Farrall - mfarrall@well.ox.ac.uk; Roger D Cox - r.cox@har.mrc.ac.uk; Mark Lathrop - mark@cng.fr; Nathalie Vionnet - vionnet@cng.fr; Marie-Thérèse Bihoreau - bihoreau@well.ox.ac.uk; Dominique Gauguier* - gdomi@well.ox.ac.uk

* Corresponding author †Equal contributors
\end{abstract}

Published: 9 July 2009

BMC Medical Genomics 2009, 2:4I doi:I0.1/86/|755-8794-2-4|

This article is available from: http://www.biomedcentral.com/I755-8794/2/4I

(c) 2009 Hu et al; licensee BioMed Central Ltd.

This is an Open Access article distributed under the terms of the Creative Commons Attribution License (http://creativecommons.org/licenses/by/2.0), which permits unrestricted use, distribution, and reproduction in any medium, provided the original work is properly cited.

\begin{abstract}
Background: Hyperglycaemia in diabetes mellitus (DM) alters gene expression regulation in various organs and contributes to long term vascular and renal complications. We aimed to generate novel renal genome-wide gene transcription data in rat models of diabetes in order to test the responsiveness to hyperglycaemia and renal structural changes of positional candidate genes at selected diabetic nephropathy (DN) susceptibility loci.

Methods: Both Affymetrix and Illumina technologies were used to identify significant quantitative changes in the abundance of over 15,000 transcripts in kidney of models of spontaneous (genetically determined) mild hyperglycaemia and insulin resistance (Goto-Kakizaki-GK) and experimentally induced severe hyperglycaemia (Wistar-Kyoto-WKY rats injected with streptozotocin [STZ]).

Results: Different patterns of transcription regulation in the two rat models of diabetes likely underlie the roles of genetic variants and hyperglycaemia severity. The impact of prolonged hyperglycaemia on gene expression changes was more profound in STZ-WKY rats than in GK rats and involved largely different sets of genes. These included genes already tested in genetic studies of $\mathrm{DN}$ and a large number of protein coding sequences of unknown function which can be
\end{abstract}


considered as functional and, when they map to DN loci, positional candidates for DN. Further expression analysis of rat orthologs of human DN positional candidate genes provided functional annotations of known and novel genes that are responsive to hyperglycaemia and may contribute to renal functional and/or structural alterations.

Conclusion: Combining transcriptomics in animal models and comparative genomics provides important information to improve functional annotations of disease susceptibility loci in humans and experimental support for testing candidate genes in human genetics.

\section{Background}

Diabetes mellitus (DM) is a growing cause of end stage renal disease in developed countries. Diabetic nephropathy (DN) develops in $35-40 \%$ of diabetic patients as the result of intrarenal metabolic, hemodynamic and structural changes $[1,2]$. DN is a complex phenotype caused by the combined effects of susceptibility alleles and environmental factors which contribute to poor glycaemic control and hypertension [3]. The importance of genetic factors in $\mathrm{DN}$ is suggested by epidemiological studies and by the familial clustering of nephropathy in both type 1 and type 2 DM [4-6]. Nephropathy does not necessarily develop in a significant proportion of diabetic patients, suggesting the involvement of specific genes.

Genetic studies of nephropathy in diabetic patients are hampered by heterogeneous clinical features, including frequent association with hypertension, and betweenstudy variation in design and phenotype assessment [7]. Although genome-wide linkage studies have identified regions of the human genome contributing to DN [8-12], the causative genes remain unknown. Association studies have been carried out with DN candidate genes that regulate blood pressure, the synthesis and degradation of renal structural components, the metabolism and transport of glucose and the process of advanced glycation [1,13,14]. High-throughput transcription profiling technologies, which are powerful tools for determining gene expression regulation in health and disease situations, can enrich genome annotations and uncover new DN candidates [14-16]. Such studies in patients and controls pose technical, scientific and ethical issues [17].

Animal models of DM are essential components of genetic and functional genomic investigations. They include animals made insulin-deficient by injection with the toxin streptozotocin (STZ) and inbred models of spontaneous DM [18]. The inbred Goto Kakizaki (GK) rat shows genetically determined alterations of glucose tolerance and insulin secretion [19], and renal structural changes similar to those observed in the early phase of $\mathrm{DN}$, including thickening of the glomerular and tubular basement membranes, and glomerular hypertrophy $[20,21]$. Elevation of glomerular macrophage infiltration is present whereas proteinuria has not been consistently observed. In contrast STZ-treated animals show little evidence of renal histopathology limited to mild glomerular mesangial expansion, which is correlated with blood glucose levels and proteinuria [22,23]. The genetic basis of these renal phenotypes in these diabetic models has not been investigated. These models underlie clearly different etiological and pathological contexts of DM, and provide complementary tools to document hyperglycaemiainduced gene expression changes that can contribute to the development of renal structural and functional anomalies relevant to DN.

In this study, we have investigated the effects of prolonged hyperglycaemia on genome-wide renal gene expression regulation in GK rats and inbred rats of similar genetic origin (Wistar-Kyoto, WKY) made severely diabetic by STZ, which may be caused by differential adaptations to mild or severe hyperglycaemia induced by genetic variants in GK and environmental changes in both diabetic models. We specifically demonstrated the possibility to use data from both comparative genomics and gene transcription regulation to test transcriptional responsiveness of genes to hyperglycaemia and renal structural changes that can improve functional annotations of known and potentially novel positional candidate genes localised in DN susceptibility loci.

\section{Methods \\ Animals}

Rats of the GK strain from the Oxford colony (GK/Ox) were used. WKY control rats were purchased from a commercial supplier (Harlan, UK). All animal procedures complied with the University of Oxford's Local Ethical Review Process and were carried out under project licences 30/2001 and 30/2324 granted by the UK Home Office. A group of 3 month old WKY rats was injected intravenously with a solution of Streptozotocin (STZ) (Sigma-Aldrich, Poole, UK) at $75 \mathrm{mg} / \mathrm{kg}$ in citrate buffer ( $\mathrm{pH} \mathrm{4.5)}$ to induce permanent and severe insulin deficiency and hyperglycaemia of about $16.6 \mathrm{mM}$. This group is referred as STZ-WKY. Experiments were performed with male rats fed standard laboratory chow pellets (B\&K Universal, Hull, UK) and kept on $12 \mathrm{~h}$ light/dark cycle. Body weight and plasma glucose were monitored. GK and WKY rats were killed at 3 months by $\mathrm{CO}_{2}$ asphyxiation, whereas 
STZ-WKY rats were killed at 6 months to ensure that duration of hyperglycaemia in both diabetic models was similar (ie. 3 months). WKY rats were used as pre STZ-treated controls for the STZ-WKY group. The left kidney was removed, snap-frozen in liquid nitrogen and stored at $80^{\circ} \mathrm{C}$ until RNA preparation. The right kidney was fixed in Dubosq-Brazil (0.4\% picric acid, $27 \%$ formalin, $7 \%$ acetic acid, 54\% ethanol) and embedded in paraffin. Sections (3 $\mu \mathrm{m}$ ) were stained with periodic acid-Schiff (PAS) reagent and counterstained with hematoxylin (Sigma-Aldrich, Poole, UK). Presence of renal anomalies in diabetic rats was evaluated by examining 3 sections per rat.

\section{RNA preparation}

Total kidney RNA samples were individually prepared from four (GK, WKY) or five (STZ-WKY) animals per group. Total RNA was extracted twice using Trizol reagents (Invitrogen Life Technologies, Paisley, UK) and cleaned with RNeasy columns (Qiagen Ltd., Crawley, UK). RNA quality was determined with an Agilent 2100 Bioanalyzer (Agilent Technologies, Waldbronn, Germany).

\section{Hybridization to Affymetrix gene expression arrays}

Synthesis of cDNA and biotin-labeled cRNA (Affymetrix ltd., High Wycombe, UK) was performed using $14 \mu \mathrm{g}$ of RNA. Biotinylated cRNA $(15 \mu \mathrm{g})$ were fragmented and individually hybridized to GeneChip Rat expression Arrays 230A containing 15,866 probe sets (Affymetrix ltd., High Wycombe, UK). Best quality samples from three animals per group were used and individually hybridized to the arrays. Washing and staining procedures were performed using a Fluidics Station 450 according to manufacturer's protocol (Affymetrix ltd, High Wycombe, UK). Arrays were scanned at $560 \mathrm{~nm}$ using an array scanner (Agilent Technologies, Waldbronn, Germany).

\section{Hybridization to Illumina gene expression arrays}

Replication experiments were carried out with Illumina arrays, a bead-based technology that is different from that of Affymetrix. Double-stranded cDNA and biotin-labelled cRNA were synthesized from RNA (300 ng) using the Illumina ${ }^{\oplus}$ TotalPrep RNA amplification it (Ambion Inc., Austin, TX). Each biotinylated cRNA (750 ng) was hybridized to prototype Sentrix ${ }^{\otimes}$ BeadChip RatRef-12_V1_Eval Whole-Genome Gene Expression arrays containing 22,636 oligonucleotides (Illumina Inc., San Diego, CA). For each rat group, individual hybridizations were performed in technical duplicates with 4 biological replicates, including the three samples previously used for the Affymetrix experiments. Following washing steps and staining with Streptavidin-Cy3, arrays were scanned on the Illumina ${ }^{\circledast}$ BeadArray Reader and the images analyzed using the Illumina BeadStudio software.

Experiments are MIAME compliant. Protocols and data are available through ArrayExpress http://www.ebi.ac.uk/ arrayexpress/ under the accession E-MEXP-1195 (Affymetrix) and E-TABM-502 (Illumina).

\section{Quantitative real time PCR}

First-strand cDNA were synthesized from total RNA with Superscript II cDNA synthesis kits (Invitrogen Gibco, Paisley, UK). QRT-PCR was performed with oligonucleotides designed to span an intron/exon boundary (see Additional file 1) using a Rotor-Gene $3000^{\mathrm{TM}}$ system (Corbett Research, Milton, UK) and QuantiTect SYBR Green PCR kits (Qiagen Ltd, Crawley, UK). Experiments were performed with biological quadruplicates (ie. the four samples used for the Illumina experiments) and technical triplicates. Analyses were performed using the Rotor-Gene software (Corbett Research, Milton, UK). Gene dosage was calculated with the standard curve generated and normalized to actin.

\section{Gene transcription data analysis}

The renal Affymetrix and Illumina gene expression datasets were previously used alongwith other rat and mouse datasets to test and optimise appropriate methods for analysis, including data extraction and normalisation procedures [24]. Analysis of Affymetrix CEL file data was performed using the Bioconductor packages in the $\mathrm{R}$ language and environment as previously described [25]. Briefly, Affymetrix data were normalized using the RMA method and the linear model for microarray data (LIMMA) package was used to assess significance between groups. For Illumina, microarray results were normalized by quantile normalization and LIMMA was used to calculate significant differences between groups using slide number as a covariate to correct for chip-specific effects. Affymetrix and Illumina datasets were used independently to carry out statistical data analyses of gene expression changes between rat groups. Cross-platform consistency of statistical significance and fold change of renal gene expression have been reported elsewhere [24]. For both Affymetrix and Illumina datasets, P-values were corrected for multiple testing using a 5\% false discovery rate as the cut-off for significance. Data from QRT-PCRbased gene transcription analysis were analysed using SPSS version 16.0 statistical package. A Bonferroni post hoc test was used to assess differences between the diabetic models and the normoglycemic WKY strain as well as between the two diabetic models. A $p$ value of less than 0.05 was considered to be statistically significant for pairwise comparisons.

\section{Results}

\section{Pathophysiological features in GK and STZ-WKY rats}

The effect of prolonged diabetes on gene expression changes was determined in situations of similar durations (3 months) of mild spontaneous (GK) or severe experimentally-induced (STZ-WKY) hyperglycaemia. Glycaemia in STZ-WKY rats was monitored to be greater than 13.5 
$\mathrm{mM}$ and not to exceed $16.6 \mathrm{mM}$ throughout of the 3 months of the experiment. Glycaemia was significantly more elevated in GK and STZ-WKY rats than in WKY controls (Additional file 2). Body weight was similar in GK and WKY rats. Prolonged severe hyperglycaemia in 6 months old STZ-WKY rats resulted in marked decreased body weight when compared to 3 months old WKY controls

The two diabetic models showed mild renal histopathological changes, which may be relevant to early pathological events in DN progression. When compared to agematched WKY (Additional file 3A), GK rats showed glomerular alterations including basement membrane thickening, matrix accumulation of hyaline, indicating mesangial extracellular matrix expansion, and increased capillary luminal volume suggesting glomerular hypertrophy (Additional files 3B3 and 3C). Tubular basement membrane thickening was observed in both collecting and distal tubules of GK sections. Following three months of severe hyperglycaemia, STZ-WKY rats exhibited glomerular hypertrophy associated with capillary loop expansion and to a lesser extend basement membrane thickening and mild mesangial expansion (Additional files 3D-F).

\section{Affymetrix-based genome-wide renal transcription profiles in diabetic rats}

Overview of gene transcription changes

Pairwise comparisons of microarray data between rat groups sharing similar genetic backgrounds of Wistar origin were performed to provide detailed information on transcriptional responses to prolonged and permanent hyperglycaemia (GK or STZ-WKY compared to WKY), as well as specific changes caused by gene variants predisposing to diabetes and possibly kidney histopathology (GK compared to WKY) and underlying hyperglycaemia severity (GK compared to STZ-WKY). Of the probe sets representing known and predicted transcripts, 252 were found differentially regulated between GK and WKY rats and over 650 between STZ-WKY and WKY, indicating a greater impact of severe hyperglycaemia on renal transcriptional changes (Figure 1). Over 800 genes were differentially expressed between STZ-WKY and GK suggesting that renal transcriptome adaptations to hyperglycaemia in these models involve different mechanisms. Furthermore, when compared to WKY, diabetic models shared transcriptional changes for only 74 genes (corresponding to 80 probesets), which generally showed the same pattern of expression regulation (Figure 2 and additional file 4). In all comparisons there were similar numbers of genes up- and down-regulated. There was no evidence of genomic clustering of differentially expressed genes (data not shown). Pathway analysis identified gene expression mechanisms either consistently affected in both diabetic models or predominantly altered in the GK or STZ-WKY rats (Additional file 5).

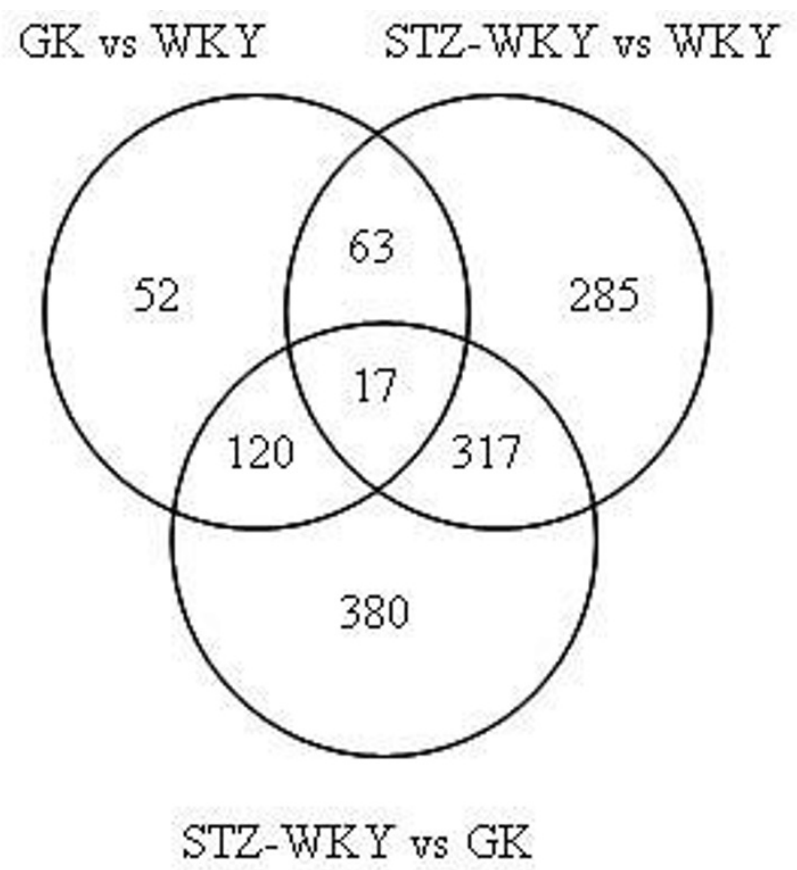

\section{Figure I}

Differentially expressed genes in kidneys of GK and STZ-induced diabetic rat models. Number of Affymetrix probesets corresponding to genes statistically differentially expressed between models $(p<0.05)$ are shown. The full list of differentially expressed genes between the three models is given in Additional file 4.

Transcription regulation of functional candidate genes in diabetic models

To gain insight into DN candidates, expression regulation of individual genes was analyzed. Evidence of significant differential expression in at least one of the comparisons between the three models was found for 1,233 Affymetrix probe sets representing 713 known genes and 468 expressed sequence tags (ESTs) and predicted genes (see Additional file 4). Owing to their relevance to mechanisms involved in DN [26], a number of these genes have already been tested for evidence of genetic association to DN $[13,27,28]$, including genes encoding the angiotensin converting enzyme (Ace), angiotensinogen (Agt), angiogenin ribonuclease A family 5 (Ang), apolipoprotein $\mathrm{E}$ (Apoe), bone morphogenetic protein 7 (Bmp7), carnosinase 1 (Cndp1), collagens type IV (Col4a1, Col4a4), connective tissue growth factor (Ctgf), cathepsin L (Ctsl), epidermal growth factor (Egf), gremlin 1 (Grem1), integrin, alpha 1 (Itga1), laminin beta 1 (Lamb1), lectin galactoside-binding soluble 3 (Lgals3), lipoprotein lipase $(L p l)$, matrix metalloproteinase $9(\mathrm{Mmp9})$, nidogen (Nid), nitric oxide synthase (Nos), neuropilin 1 (Nrp1), renin (Ren), SA hypertension-associated homolog (Sah), glucose transporter 2 (Slc2a2), a sodium/chloride transporter (Slc12a3), hepatic transcription factor 2 (Tcf2, Hnf1b1), 


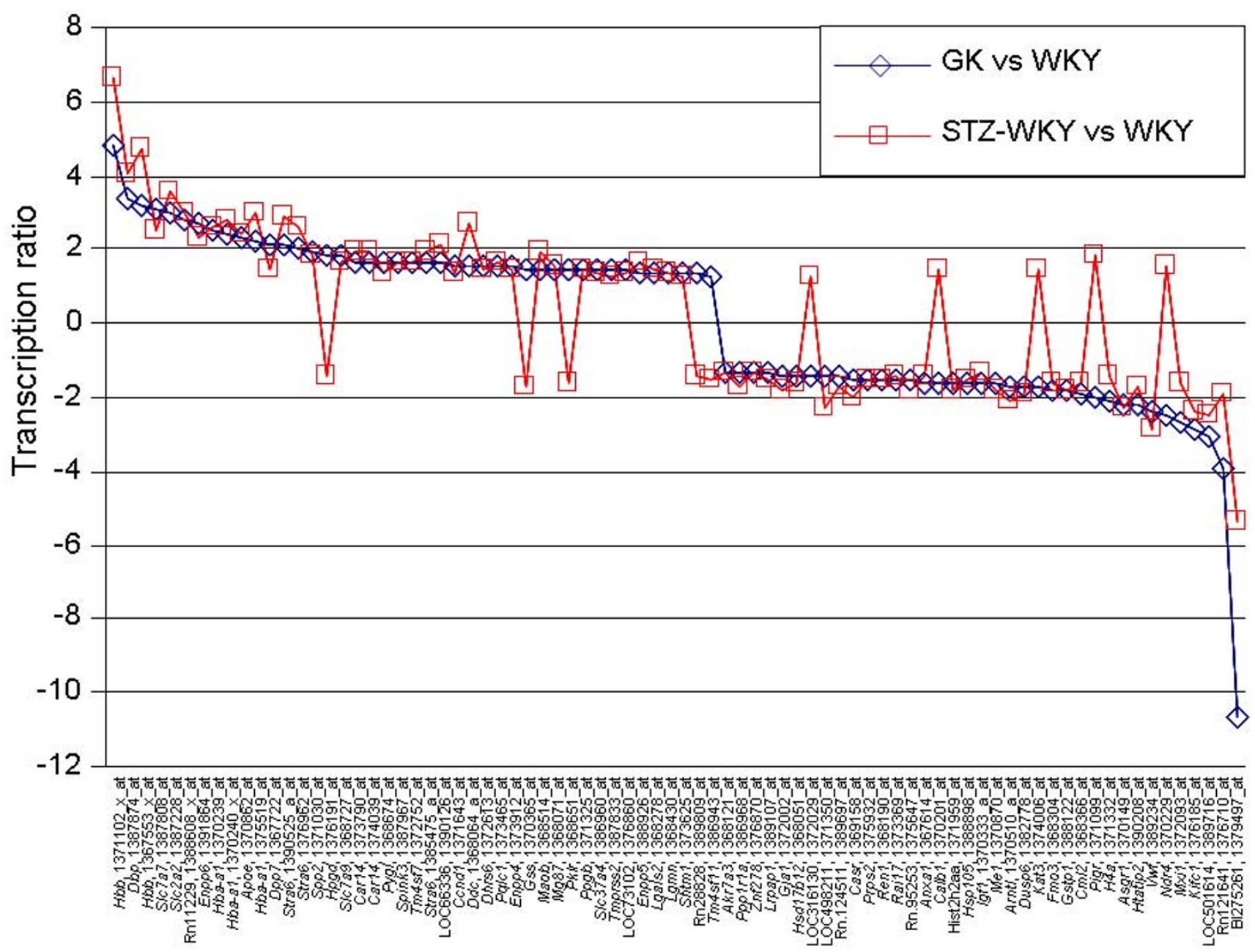

Figure 2

Patterns of transcription for genes consistently differentially expressed between diabetic rats (GK and STZWKY) and WKY controls. Genes are listed along the $X$ axis with both Affymetrix probe set names and either symbols for known genes or accession number references for EST sequences. Genes are ranked from the highest (left) to the lowest (right) changes in transcription ratio in the GK vs WKY comparison.

transcobalamin 2 (Tcn2), transforming growth factor beta 1 -induced transcript $4(\mathrm{Tg} f b 1$ i4) and transmembrane 4 superfamily member $4(T m 4 s f 4)$. Related genes and other candidates previously tested in DN genetics [13] were found differentially expressed between models, including genes encoding cathepsins (Ctsb, Ctsf), carboxypeptidases (Cpe, Cpn1), methylenetetrahydrofolate dehydrogenase (Mthfd1) and syndecan 1 ( $S d c 1)$, which were consistently upregulated in STZ-WKY when compared to WKY, and genes for carnosinase 2 (Cndp2), ectonucleotide phosphodiesterase 5 (Enpp5), laminin alpha (Lama1), betagalactoside binding lectins (Lgals1, Lgals2, Lgals5, Lgals3bp), matrix metalloproteinases (Mmp11, Mmp14) and a matrix-associated regulator of chromatin (Smarca4).
Altered transcription regulation between models was also found for genes involved in extracellular matrix turnover (Cdh1, Cdh16, Cdh17, Celsr2, Cyr61, Dag1, Mucdhl, Omd, Sparc, Spon1) and biochemical processes including the polyol pathway (Akr1b4, Akr1b8, Akr1c12, Akr7a3, Sord) and gluthatione metabolim (Gss, Gstm1, Gsto1, Gstp1, Gstt2, Hagh, Yc2) (Additional file 4) which are relevant to DN. However, nearly $40 \%$ of genes differentially expressed between diabetic rats correspond to ESTs and predicted genes, which lack unambiguous annotation but represent attractive DN candidates, in particular when they map to rat chromosomal regions conserved with DN susceptibility loci. 


\section{Replication of gene expression changes using Illumina BeadChips}

To evaluate the robustness of transcription changes detected with Affymetrix arrays, gene expression profiling was repeated with the same samples using a different technology (Illumina BeadChips). Technical aspects of Affymetrix and Illumina array data processing (signal extraction and normalisation) and cross platforms consistency of results in terms of fold-change of expression and statistical significance, have been previously addressed [24]. Of the 1,182 transcripts found differentially regulated in at least one of the comparisons (714 known genes and 468 ESTs), over 750 could be unambiguously identified on the Illumina array, including $80 \%$ of known genes (566 genes) and 41\% of ESTs (193 ESTs) (Additional file 6). In all comparisons, replication of Affymetrix results with Illumina in terms of both statistical significance of differential expression and direction of transcriptional change was remarkably high for known genes, ranging from $83 \%$ to $90 \%$, whereas replication rate for ESTs was more modest (56\% to 70\%). Lower replication rate of Affymetrix results by Illumina for ESTs than known genes may reflect ambiguous annotations of ESTs. In exceptional cases (Dpt, Hmox2, Pde4d, Slc12a3, Stat3, $W d f y 1$ and 7 ESTs) the directions of expression changes given by Illumina and Affymetrix were inconsistent. Large numbers of genes were found differentially expressed with Illumina but not Affymetrix despite very similar expression ratio. This can be explained by the use of technical replicates for the Illumina experiments rather than technical features of the platforms.

Identification of DN functional and positional candidates To study more specifically expression of genes localized in DN susceptibility loci, we initially used comparative genome mapping data [29] to determine that regions of rat chromosomes $2 q 24-25,2 q 31-32,3 q 36-43,8 q 12-13$, 8q31-32, 11q22-23, 13p12-13, 18p12-13 and 18q12-13 show evidence of synteny conservation with DN loci in human 3q23-q29, 7q32.3-q33, 18q22-q23 and 20p12.3p13. Using EnsEmbl genome annotation and the EnsMart browser http://www.ensembl.org/, we selected positional candidate genes and predicted protein coding sequences at these loci to investigate their expression regulation in the Affymetrix datasests. There are over 330 annotated genes and ESTs at these loci, of which over 210 (65\%) are represented on the Affymetrix array. A total of $34 \mathrm{DN}$ positional candidates were differentially expressed in at least one of the comparisons between diabetic rats and controls (Table 1)(Additional file 7). These include candidates already tested in DN genetic studies (Cndp1, Slc2a2) and genes known to be exclusively or abundantly expressed in kidney, such as genes encoding enzymes of the polyol pathway (Akr1b4,Akr1b8), a kidney specific urea transporter (Slc14a2), a component of renal tight junctions (Cldn16), a zinc metalloendopeptidase (Mep1b) and a marker of tubular injury ( $R b p 1)$. Altered transcription of other genes at the loci underlines their possible role in DN etiology and pathogenesis (Table 1).

To provide information on renal expression of DN positional candidates not represented on the Affymetrix array, 53 genes localized in the most significant region of DN loci were selected for quantitative real time PCR (QRTPCR) on kidney samples of diabetic and control rats. Rat orthologs of 23 of these could not be identified using EnsMart. Three genes (AGBL3, CHCHD3, LRGUK) and two ESTs (Q68DL7, Q6ZU70) are unlikely to be expressed in kidney as the oligonucleotides for the rat orthologs failed to amplify renal cDNA. Of the remaining 25 genes, 16 showed evidence of differential expression between diabetic and control rats (Table 2). With the exception of an aldo-keto reductase (Akr1b10), the function of these genes is largely unknown and their involvement in pathological mechanisms described in DN has not been reported.

Overall, results from transcriptional analysis of DN positional candidates in the kidney of diabetic rats provided novel functional annotations (ie. transcriptional adaptations to hyperglycaemia and renal structural changes) of known and predicted genes.

\section{Validation of microarray data by QRT-PCR}

To further validate transcription changes derived by microarray platforms, QRT-PCR was carried out with 12 genes selected for their functional relevance to DN $(B m p 3$, Bmp6, Grem1, Ctgf, Slc2a2) or Type 2 DM (Arntl, Slc2a2), their location in DN susceptibility loci (Cldn16, Hrg, Pld1, $R b p 1, S l c 2 a 2)$ or the magnitude of the transcriptional change (JunD, Slc2a2, Tff3) (Figure 3). Results generally confirm microarray data including the direction of expression changes. Downregulation of Bmp3 expression in GK and upregulation of Grem1 in STZ-WKY confirm Illumina results.

\section{Discussion}

We report renal genome-wide gene expression changes induced by prolonged hyperglycaemia in models of experimentally-induced (STZ-WKY) or spontaneous (GK) diabetes, widely used in diabetes research and known to develop renal alterations $[18,20,30]$. Two well-established transcription profiling technologies (Affymetrix and Illumina) provided highly concordant information on the effects of severe (STZ-WKY) or moderate (GK) hyperglycaemia, as well as GK-specific diabetes susceptibility genes, on the expression of over 15,000 known and predicted genes. Differentially expressed genes, which reflect mechanisms responsive to hyperglycaemia, provide improvements in functional annotations of known and predicted genes and potentially novel targets in DN genetics. 
Table I: Genes differentially expressed between diabetic models and controls that map to regions conserved with human DN susceptibility loci.

\begin{tabular}{|c|c|c|c|c|c|c|c|c|}
\hline \multirow[b]{2}{*}{ Gene Symbol } & \multirow[b]{2}{*}{ Gene description } & \multirow[b]{2}{*}{ Genbank } & \multicolumn{2}{|c|}{ GK vs WKY } & \multicolumn{2}{|c|}{ STZ vs WKY } & \multicolumn{2}{|c|}{ STZ vs GK } \\
\hline & & & $\mathrm{TR}$ & $\mathrm{P}$-value & TR & $\mathrm{P}$-value & $\mathrm{TR}$ & $\mathrm{P}$-value \\
\hline \multicolumn{9}{|c|}{ Human 3q23-q29 } \\
\hline Bdh & 3-hydroxybutyrate dehydrogenase & NM 053995 & +1.3 & Ns & -1.2 & Ns & -1.6 & 0.007 \\
\hline Centb2 & Centaurin beta 2 & BM389190 & -1.4 & Ns & 1.0 & Ns & +1.3 & 0.047 \\
\hline Clcn2 & Chloride channel 2 & NM 017137 & +1.2 & Ns & -1.1 & Ns & -1.4 & 0.038 \\
\hline Cldn 16 & Claudin 16 & NM_131905 & -1.1 & Ns & -1.6 & 0.002 & -1.4 & 0.01 \\
\hline Dgkg & Diacylglycerol kinase gamma & NM 013126 & +1.2 & Ns & +1.6 & 0.025 & +1.3 & Ns \\
\hline Dnajb II & DnaJ (Hsp40) subfamily BII & $\underline{B} 1295873$ & 1.0 & Ns & -1.3 & 0.043 & -1.3 & Ns \\
\hline Eif4gI & Eukaryotic translation initiation factor & $\underline{\mathrm{BC} 098868}$ & +1.1 & Ns & -1.2 & Ns & -1.4 & 0.011 \\
\hline FadI04 & FADI04 (predicted) & All 76320 & +1.3 & Ns & -1.3 & 0.05 & -1.7 & $5 \times 10^{-4}$ \\
\hline Fam43a & Family with sequence similarity $43 \mathrm{~A}$ & $\underline{\mathrm{BEI} 08405}$ & -1.1 & Ns & -1.7 & 0.017 & -1.5 & Ns \\
\hline $\mathrm{Hrg}$ & Histidine-rich glycoprotein & NM 133428 & -1.2 & Ns & +2.0 & 0.034 & +2.5 & 0.007 \\
\hline \multirow[t]{2}{*}{$M f n l$} & Mitofusin I & All69627 & 1.0 & Ns & +1.7 & 0.046 & +1.7 & 0.035 \\
\hline & & $\underline{\text { AA943135 }}$ & 1.0 & Ns & +1.5 & 0.018 & +1.5 & 0.009 \\
\hline Ncbp2 & Nuclear cap binding protein subunit 2 & $\underline{B} 1282103$ & +1.3 & Ns & -1.5 & 0.046 & -2 & 0.002 \\
\hline PIdI & Phospholipase DI & $\underline{\mathrm{U} 69550}$ & -1.3 & Ns & -1.8 & 0.036 & -1.4 & Ns \\
\hline Pigx & Subunit of GPI-mannosyltransferase & All72192 & +1.3 & Ns & 1.0 & Ns & -1.3 & 0.043 \\
\hline Rbpl & Retinol binding protein I & NM_012733 & 1.0 & Ns & +2.3 & $3 \times 10^{-4}$ & +2.2 & $3 \times 10^{-4}$ \\
\hline Siat 1 & Sialyltransferase I & $M 83143$ & 1.0 & Ns & -1.6 & 0.002 & -1.6 & 0.001 \\
\hline Slc2a2 & Solute carrier family 2 member 2 (Glut2) & NM 012879 & +3.0 & $5 \times 10^{-4}$ & +3.6 & $1 \times 10^{-4}$ & +1.2 & Ns \\
\hline Ssr3 & Signal sequence receptor gamma & NM 031120 & -1.2 & Ns & +1.3 & Ns & +1.6 & 0.003 \\
\hline Zbed4 & Zinc finger, BED domain containing 4 & Al170289 & -1.1 & Ns & -1.3 & 0.046 & -1.2 & Ns \\
\hline--- & Similar to protein NP 079596 & $\underline{A l 412011}$ & +1.4 & Ns & -1.5 & 0.044 & -2.1 & 0.001 \\
\hline \multicolumn{9}{|c|}{ Human 7q32.3-q33 } \\
\hline Akrlb4 & Aldo-keto reductase family IB4 & NM 012498 & 1.0 & Ns & +2.2 & Ns & +2.1 & 0.024 \\
\hline Akrlb8 & Aldo-keto reductase family IB8 & NM 173136 & -6.2 & $6 \times 10^{-5}$ & +1.4 & Ns & +8.5 & $8 \times 10^{-6}$ \\
\hline Slc35b4 & Solute carrier family 35 member B4 & XM 216122 & +1.2 & Ns & -1.1 & Ns & -1.4 & 0.010 \\
\hline \multicolumn{9}{|c|}{ Human $18 q 12-q 23$} \\
\hline Acaa2 & Acetyl-Coenzyme $A$ acyltransferase 2 & NM 130433 & -1.6 & 0.041 & -1.3 & Ns & +1.2 & Ns \\
\hline Brunol4 & RNA binding protein bruno-like 4 & $\overline{\mathrm{AW} 530502}$ & +1.1 & Ns & +1.3 & 0.036 & +1.2 & Ns \\
\hline Cndpl & Carnosinase I & $\mathrm{Al} 231438$ & 1.0 & Ns & -3.4 & $3 \times 10^{-5}$ & -3.5 & $8 \times 10^{-6}$ \\
\hline Cndp2 & Carnosinase 2 & $\underline{\mathrm{B}} 279729$ & +1.1 & Ns & -1.3 & 0.042 & -1.4 & 0.01 \\
\hline GOS2 & Putative lymphocyte G0/GI switch gene & Al406939 & +1.3 & Ns & -1.6 & Ns & -2.1 & 0.004 \\
\hline Mapk4 & Mitogen-activated protein kinase 4 & BG378232 & +1.3 & Ns & +1.5 & 0.023 & +1.2 & Ns \\
\hline Meplb & meprin I beta (endopeptidase-2) & NM 013183 & 1.0 & Ns & -1.4 & 0.042 & -1.4 & 0.050 \\
\hline Pqlel & PQ loop repeat containing I & Al228284 & +1.6 & 0.044 & +1.7 & 0.011 & +1.1 & Ns \\
\hline \multirow[t]{2}{*}{ SIc $14 a 2$} & Solute carrier family 14 , member 2 & NM 019347 & -1.1 & Ns & +1.5 & 0.020 & +1.7 & 0.005 \\
\hline & & $\mathrm{AF042167}$ & -1.1 & Ns & +1.4 & Ns & +1.6 & 0.035 \\
\hline \multicolumn{9}{|c|}{ Human 20p I 2.3-p /3 } \\
\hline$c d c 25 b$ & Cell division cycle 25 homolog B & NM 133572 & +1.2 & Ns & +1.6 & 0.028 & +1.4 & Ns \\
\hline Prnp & Prion protein & NM 012631 & +1.6 & 0.029 & +1.1 & Ns & -1.5 & 0.030 \\
\hline
\end{tabular}

Gene expression results from Affymetrix chip hybridizations are given as transcription ratio (TR). The list of genes in these regions represented on the Affymetrix chip and not differentially expressed is given in Additional file 7. Ns, not statistically significant differences.

Renal histological changes in GK and STZ-WKY rats are relatively mild in these models [21,30-32], which may therefore be useful for investigating early molecular adaptations to hyperglycaemia in DN progression. Genomewide gene expression profiling, which allows unbiased analysis of thousands of genes regardless of their function and chromosomal position, is a practical approach to comprehensively assess perturbed mechanisms in GK and STZ-WKY rats maintained in strictly identical conditions and uncover renal molecular adaptations to impaired glucose homeostasis in a context where environmental influ- ences and genetic polymorphisms are minimized, as inbred WKY and GK strains share extensive genetic similarities outside GK-specific diabetes variants $[19,32]$. Gene expression results from two well-established and robust microarray technologies designed to investigate the transcription of largely overlapping series of genes [24], which provide systems for assessing data replication, showed remarkable concordance in transcription regulation patterns. Some discordant results may be explained by the design of the probesets (Affymetrix) or the oligonucleotides (Illumina) in different gene isoforms or the 
Table 2: Expression regulation of rat orthologs of human genes localised in DN susceptibility loci and not represented on the Affymetrix arrays.

\begin{tabular}{|c|c|c|c|c|c|c|c|c|c|}
\hline \multirow{2}{*}{$\begin{array}{l}\text { Human Ensembl } \\
\text { (ENSG00000) }\end{array}$} & \multirow[b]{2}{*}{ Description } & \multirow[b]{2}{*}{ Symbol } & \multirow[b]{2}{*}{$\begin{array}{l}\text { Rat Ensembl } \\
\text { (ENSRNO G000000) }\end{array}$} & \multicolumn{2}{|c|}{ GK vs WKY } & \multicolumn{2}{|c|}{ STZ-WKY vs WKY } & \multicolumn{2}{|c|}{ STZ-WKY v GK } \\
\hline & & & & TR & p-value & TR & p-value & TR & p-value \\
\hline \multicolumn{10}{|l|}{ Human 3q23 } \\
\hline 175066 & Glycerol kinase 5 & GK5 & 10942 & 1.00 & Ns & 0.66 & 0.026 & 0.67 & $2 \times 10^{-3}$ \\
\hline 114120 & $\begin{array}{l}\text { Solute carrier family } 25 \text {, } \\
\text { member } 36\end{array}$ & SLC25A36 & 39085 & 1.28 & Ns & 1.54 & $4 \times 10^{-3}$ & 1.21 & Ns \\
\hline 175093 & $\begin{array}{l}\text { SPRY domain-containing } \\
\text { SOCS box protein } 4\end{array}$ & SPSB4 & 12862 & 0.78 & Ns & 0.37 & $5 \times 10^{-3}$ & 0.47 & 0.013 \\
\hline 114126 & $\begin{array}{l}\text { Transcription factor Dp-2 } \\
\text { (E2F dimerization partner 2) }\end{array}$ & TFDP2 & $|124|$ & 0.76 & Ns & 1.56 & Ns & 2.04 & Ns \\
\hline 114127 & $5^{\prime}-3^{\prime}$ exoribonuclease I & XRNI & 10027 & 0.71 & Ns & 0.66 & 0.030 & 0.93 & Ns \\
\hline$|773| 1$ & $\begin{array}{l}\text { Zinc finger and BTB domain- } \\
\text { containing protein } 38\end{array}$ & ZBTB38 & 12386 & 1.08 & Ns & 1.27 & 0.026 & 1.17 & Ns \\
\hline 206250 & Protein coding sequence & - & 22555 & 0.81 & Ns & 0.82 & Ns & 1.01 & Ns \\
\hline \multicolumn{10}{|l|}{ Human 7q33 } \\
\hline 146856 & $\begin{array}{l}\text { ATP/GTP binding } \\
\text { protein-like } 3\end{array}$ & AGBL3 & 10397 & 0.95 & Ns & 0.52 & $1 \times 10^{-4}$ & 0.55 & $3 \times 10^{-4}$ \\
\hline 198074 & $\begin{array}{l}\text { Aldo-keto reductase I } \\
\text { member BI0 }\end{array}$ & AKRIBIO & 27433 & 1.94 & 0.035 & 0.77 & Ns & 0.40 & $1 \times 10^{-4}$ \\
\hline 122783 & $\begin{array}{l}\text { Uncharacterized protein } \\
\text { C7orf49 }\end{array}$ & C7orf49 & 26958 & 0.95 & Ns & 0.59 & 0.016 & 0.62 & $5 \times 10^{-3}$ \\
\hline \multicolumn{10}{|c|}{ Human $18 q 22.3-23$} \\
\hline $17998 \mid$ & Teashirt zinc finger homeobox & TSHZI & 16246 & 0.91 & Ns & 1.00 & Ns & 1.10 & Ns \\
\hline 141665 & F-box only protein 15 & FBXOI5 & 38225 & 0.72 & Ns & 0.69 & Ns & 0.97 & Ns \\
\hline 130856 & Zinc finger protein 236 & ZNF236 & 16303 & 1.45 & 0.025 & 1.48 & 0.011 & 1.02 & Ns \\
\hline 166540 & zinc finger protein 407 & ZNF407 & 16008 & 0.87 & Ns & 0.77 & 0.015 & 0.88 & Ns \\
\hline 101493 & Zinc finger protein 516 & ZNF5I6 & 16258 & 1.07 & Ns & 1.20 & Ns & 1.12 & Ns \\
\hline \multicolumn{10}{|c|}{ Human $20 \mathrm{p} / 2.3-13$} \\
\hline |4945| & $\begin{array}{l}\text { Disintegrin and } \\
\text { metalloproteinase } 33\end{array}$ & ADAM33 & 21242 & 0.85 & Ns & 1.28 & Ns & 1.51 & Ns \\
\hline 101222 & $\begin{array}{l}\text { Uncharacterized protein } \\
\text { C20orf28 }\end{array}$ & C20orf28 & 21247 & 1.19 & Ns & 0.80 & Ns & 0.67 & 0.016 \\
\hline 101311 & $\begin{array}{l}\text { Unc-I I 2-related protein I } \\
\text { (Kindlin-I) }\end{array}$ & C20orf42 & 21274 & 0.70 & 0.050 & 0.53 & $2.2 \times 10^{-3}$ & 0.75 & Ns \\
\hline 125872 & $\begin{array}{l}\text { Uncharacterized protein } \\
\text { C20orf75 }\end{array}$ & C20orf75 & 32989 & 1.65 & Ns & 5.48 & $1 \times 10^{-3}$ & 3.31 & $1 \times 10^{-3}$ \\
\hline 171984 & $\begin{array}{l}\text { Uncharacterized protein } \\
\text { C20orfl96 }\end{array}$ & C20orfl96 & 21268 & 1.40 & Ns & 1.22 & Ns & 0.87 & Ns \\
\hline 125885 & $\begin{array}{l}\text { Minichromosome maintenance } \\
\text { complex component } 8\end{array}$ & MCM8 & 21272 & 2.63 & Ns & 2.82 & Ns & 1.08 & Ns \\
\hline 171864 & Prion protein 2 & PRND & 21260 & 0.64 & Ns & 0.53 & Ns & 0.83 & Ns \\
\hline 101236 & RING finger protein 24 & RNF24 & 21250 & 0.81 & Ns & 0.60 & 0.010 & 0.74 & Ns \\
\hline 088827 & $\begin{array}{l}\text { Sialic acid-binding Ig-like } \\
\text { lectin I }\end{array}$ & SIGLECI & 21243 & 0.73 & 0.033 & 0.81 & Ns & 1.11 & Ns \\
\hline 149345 & $\begin{array}{l}\text { Putative ubiquitin-conjugating } \\
\text { enzyme E2 D3-like }\end{array}$ & U2D3L & $|374|$ & 1.27 & Ns & 0.64 & 0.020 & 0.51 & $2 \times 10^{-3}$ \\
\hline
\end{tabular}

The EnsMart browser http://http:www.ensembl.org/ was used to identify human-rat homologies (synteny conservation) in regions of the human genome associated with DN. Transcription ratios (TR) between models were assessed by QRT-PCR with kidney samples of GK, STZ-WKY and WKY rats previously used to hybridize microarrays. Ns, not statistically significant.

existence of polymorphisms in the sequences arrayed on the chips [33].

Transcriptomic alterations were more profound in STZWKY than in GK and involved largely non-overlapping sets of genes, thus underlining the importance of investigating gene expression in very different, but complementary, contexts of diabetes aetiology and pathogenesis. Age may have a confounding impact on these processes as, for obvious reasons, the models used were not age-matched, but the duration of spontaneous (GK) or experimentallyinduced (STZ-WKY) hyperglycaemia was identical (3 months). Along the same line, gene expression differences between STZ-WKY and WKY, which compare pre- and post-drug treated groups and test molecular responses to prolonged and severe hyperglycaemia, may also reflect to 


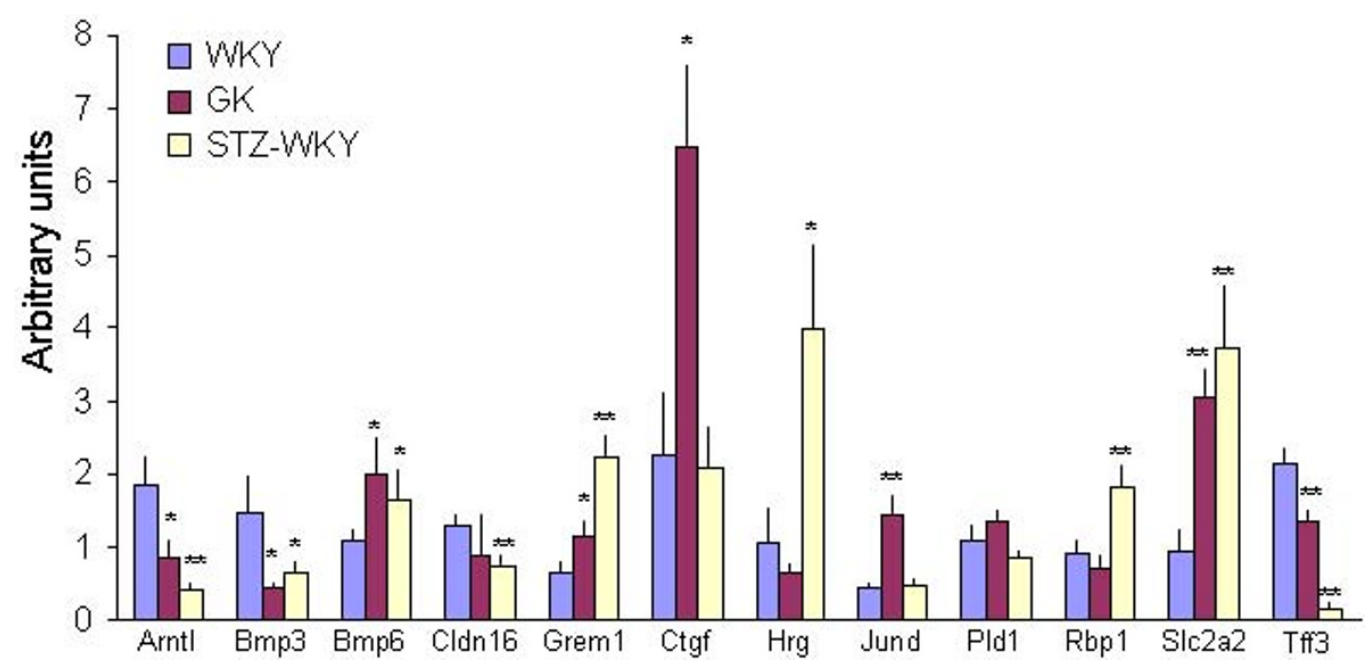

Figure 3

Validation of microarray results by quantitative real time PCR. The expression of 12 selected genes was assessed by QRT-PCR in kidney samples of GK, STZ-WKY and WKY rats previously used for microarray-based transcription profiling. Results are expressed as means $\pm \mathrm{SE}$, in percentage of normalized $\mathrm{WKY}$ values. ${ }^{*} \mathrm{P}<0.05$, $* * \mathrm{P}<0.0$ I statistically significantly different to WKY controls.

some extent "normal" biological adaptations to ageing described in WKY [34]. Similar model-specific patterns of gene expression regulation was observed in renal transcriptomes of mouse models of STZ-induced and spontaneous $(d b / d b)$ diabetes [31]. Diabetes-predisposing variants in the GK and $d b / d b$ strains may therefore affect renal changes through specific mechanisms not necessarily related to hyperglycaemia itself. The existence of such specific polymorphisms [35] and a genetic predisposition to salt-induced hypertension [36] are documented in the GK strain. Metabolic and hormonal factors, as well as renal structural alterations secondary to impaired glucose regulation, may also explain the extent of renal transcriptomic differences between STZ-WKY and GK. Insulin deficiency is the leading cause of hyperglycaemia in STZtreated animals [37], whereas the GK strain develops insulin resistance, which is associated with DN [38] and may play a specific role on renal gene expression in GK rats.

Combining transcriptomic results in GK and STZ-WKY rats with published data in animal models of diabetes $[31,39,40]$. and in humans $[41,42]$ contribute to the enrichment of renal functional pathways that may be involved in DN. Of note, upregulation of Grem1, a bone morphogenetic protein antagonist, and strong downregulation of cadherins, Egf and Tff3, a protein promoting epithelial cell restitution, may contribute to renal damage in STZ-WKY, $[43,44]$. In GK rats, altered transcription of proliferation and differentiation factors (Bmp3, Bmp6, Bmp7) [45] suggests the involvement of mechanisms protecting the kidney against interstitial fibrosis [20], that upregulation of transcripts increasing extracellular matrix produc- tion (Ctgf, Mmp9) may initiate [46]. Downregulation of aldo-keto reductases $(A k r 1 b 8, A k r 1 c 12, A k r 7 a 3)$ and upregulation of Sord (in STZ-WKY) may reflect the activation of compensatory mechanisms preventing the accumulation of sorbitol and reducing polyol pathway flux [26]. Stimulated expression of glutathione synthase in GK may reflect cytoprotective mechanisms, whereas its downregulation in STZ-WKY may be balanced by increased expression of glutathione transferases, therefore contributing to protect the cells against oxidative stress [47]. However, expression of genes encoding protein known to play a role in DN pathogenesis, including TGFB, heme oxygenase (HMOX1), osteopontin (SPP1), were not significantly altered in diabetic rats, despite a $30-40 \%$ upregulation.

The primary aim of the study was to identify genes that are differentially expressed in diabetic models and map to regions of the rat genome that show evidence of synteny conservation with human loci associated with DN. As largely overlapping series of genes are represented on Illumina and Affymetrix arrays, replicated gene transcription differences with the two technologies provide robust information for selecting positional and functional DN candidate genes. Candidates include genes and ESTs that have no apparent functional relevance to DN, but their renal transcription patterns indicate a possible role in kidney structural and metabolic alterations. Of note, hepatic transcription profiling data in the same animals show that only a small proportion $(6 \%-11 \%)$ of genes are consistently differentially expressed in kidney and liver (data not shown). Comparative genomic analysis of $\mathrm{DN}$ positional 
candidates mapped to $3 \mathrm{q}, 7 \mathrm{q}, 18 \mathrm{q}$ and $20 \mathrm{p}$ [8-11] revealed several differentially expressed genes that have not been tested in genetic studies of DN $[13,27,28]$ and may therefore be important in genetic and clinical investigations of DN. Among relevant genes, Slc14a2 encodes a vasopressin-dependent urea transporter expressed in the collecting duct. Its upregulation in STZ-WKY may contribute to nitrogen conservation in response to glucosurea [48]. In these rats, downregulation of Cldn16, a major structural component of tight junction in the ascending limb of Henle, may result in ions wasting and renal glomerular and tubular alterations [49,50]. Finally, altered renal transcription of Rbp1 and Mep $1 b$ has been reported in mouse models of experimentally-induced diabetes [31] and tubular fibrosis [51].

Positional candidates differentially expressed in diabetic rats also include protein coding sequences predicted by bioinformatic models which nevertheless represent important functional DN candidates. Even though genome annotations and microarray technologies keep improving, potentially important DN positional candidate genes are not represented on gene expression arrays. Systematic renal expression analysis of ESTs mapped to the most significant regions of DN loci identified transcripts differentially regulated between GK and STZ-WKY rats. Of note, using quantitative RT-PCR, we found evidence of significant changes in expression for transcripts predicted to encode a further aldo-keto reductase (Akr1b10) and the zinc finger protein 236, which has been tested as a DN candidate [52]. In the vast majority of cases, confirmation of gene organization and biological functions, including altered protein abundance, is required. Although we prioritized our study to gene expression studies in total kidney to take into account interactions between renal cell types in the regulation of organ function, further investigations in tubular and mesangial cells will establish tissue specific gene expression patterns.

\section{Conclusion}

Our study illustrates a pertinent approach utilizing both functional and positional criteria for selecting disease candidate genes. It provides a comprehensive survey of differential renal transcription adaptations to prolonged severe or moderate hyperglycaemia which likely involve pathophysiological and compensatory mechanisms. Comparative genomic analysis of genome-wide gene expression data can be used to initiate human genetic studies on specific genes in that may provide entry points in etiologically important gene pathways in DN.

\section{Abbreviations}

DM: Diabetes Mellitus; DN: Diabetic Nephropathy; EST: expressed sequence tag; GK: Goto-Kakizaki; IPA: Ingenu- ity Pathway Analysis; LIMMA: Linear Models for Microarray Analysis; RMA: Robust Multi-array Analysis; STZ: Streptozotocin; WKY: Wistar-Kyoto; QRT-PCR: quantitative real time PCR; TR: transcription ratio.

\section{Competing interests}

The authors declare that they have no competing interests.

\section{Authors' contributions}

YH, PYW and MTB performed comparative genomic analysis and qRT-PCR. CB and MTB performed Affymetrixbased gene transcription profiling. PJK and KA performed Illumina-based gene transcription studies. KA and KJW carried out phenotypic and histological analyses of the rat models. SPW and MTB carried out statistical analyses, bioinformatic studies, gene annotations of microarray data and biological data interpretation. MTB, NV and DG drafted the manuscript. LT, PHG, SH, MM, HHP, MF, RDC, ML, NV, MTB and DG conceived of the study, and participated in its design and coordination. All authors read and approved the final manuscript.

\section{Additional material}

\section{Additional file 1}

Oligonucleotides designed for QRT-PCR analysis of renal gene transcription regulation in diabetic and control rats. Details of symbols, descriptions and GenBank accession numbers of genes tested for differential expression between GK, STZ-WKY and WKY rats using QRT-PCR, alongwith primer sequence and PCR product length.

Click here for file

[http://www.biomedcentral.com/content/supplementary/17558794-2-41-S1.xls]

\section{Additional file 2}

Phenotypic features of GK, STZ-WKY and WKY rats. Body weight and plasma glucose concentrations in GK, STZ-WKY and WKY rats. Click here for file

[http://www.biomedcentral.com/content/supplementary/17558794-2-41-S2.doc]

\section{Additional file 3}

Photomicrographs of PAS-stained kidney sections from GK, STZ-WKY and WKY rats $(\times 400)$. Renal histopathological features in the diabetic strains (GK, STZ-WKY) and in WKY normoglycaemic controls. Click here for file

[http://www.biomedcentral.com/content/supplementary/17558794-2-41-S3.tiff]

\section{Additional file 4}

Details of genes differentially expressed in kidneys of GK and STZWKY models of diabetes and WKY controls. Details of Affymetrix probeset references, symbols and descriptions of genes found significantly differentially expressed $(P<0.05)$ between diabetic rats $(G K, S T Z-W K Y)$ and WKY controls, alongwith calculation of transcription fold change and statistical significance of transcriptional changes.

Click here for file

[http://www.biomedcentral.com/content/supplementary/17558794-2-41-S4.xls] 


\section{Additional file 5}

Overview of renal transcriptomic changes in pathways underlying differential gene expression adaptations to moderate or severe hyperglycaemia in rat models of diabetes. Selection of the most significant functionally related groups of genes found differentially expressed between diabetic rats (GK, STZ-WKY) and WKY controls.

Click here for file

[http://www.biomedcentral.com/content/supplementary/17558794-2-41-S5.doc]

\section{Additional file 6}

Comparison of Affymetrix and Illumina kidney gene expression profiling data for genes found differentially expressed between GK, STZWKY and WKY rats using the Affymetrix platform. Replication analysis of Affymetrix-based gene expression changes between GK, STZ-WKY and WKY rats using data from Illumina arrays showing fold-changes and statistical significance of differential expression derived by the two microarray technologies.

Click here for file

[http://www.biomedcentral.com/content/supplementary/17558794-2-41-S6.xls]

\section{Additional file 7}

Genes mapped to chromosomal regions of the rat genome conserved with human DN loci and found not differentially expressed between diabetic models. Details of Affymetrix probeset references, symbols and descriptions of rat genes localised in genomic regions homologous to human DN susceptibility loci, and not significantly differentially expressed $(P>0.05)$ between diabetic rats $(G K, S T Z-W K Y)$ and WKY controls, alongwith calculation of transcription fold change and statistical significance of transcriptional changes.

Click here for file

[http://www.biomedcentral.com/content/supplementary/17558794-2-41-S7.xls]

\section{Acknowledgements}

This work was supported by the Wellcome Trust Functional Genomics Initiative CFG (Cardiovascular Functional Genomics, 066780) and grants from the European Commission (EURAGEDIC, QLRT-2000-01669 and FGENTCARD, LSHG-CT-2006-037683). The authors acknowledge support of the Wellcome Trust Functional Genomics Initiative BAIR (Biological Atlas of Insulin Resistance, 066786) for microarray data analysis. Y Hu is a recipient of an Albert Renold fellowship of the European Foundation for the Study of Diabetes. SP Wilder was supported by a Wellcome Prize Studentship in Bioinformatics and Statistical Genetics. D Gauguier holds a Wellcome Senior Fellowship in Basic Biomedical Science (057733).

\section{References}

I. Wolf G: New insights into the pathophysiology of diabetic nephropathy: from haemodynamics to molecular pathology. Eur J Clin Invest 2004, 34( I 2):785-796.

2. Rossing P: Prediction, progression and prevention of diabetic nephropathy. The Minkowski Lecture 2005. Diabetologia 2006 , 49(I): II-19.

3. Group TDCaCTR: Clustering of long-term complications in families with diabetes in the diabetes control and complications trial. Diabetes 1997, 46(II):1829-1839.

4. Krolewski AS: Genetics of diabetic nephropathy: evidence for major and minor gene effects. Kidney Int 1999, 55(4): 1582-1596.

5. Seaquist ER, Goetz FC, Rich S, Barbosa J: Familial clustering of diabetic kidney disease. Evidence for genetic susceptibility to diabetic nephropathy. N Engl J Med 1989, 320( 18): I |6|-| |65.
6. Imperatore G, Knowler WC, Pettitt DJ, Kobes S, Bennett PH, Hanson RL: Segregation analysis of diabetic nephropathy in Pima Indians. Diabetes 2000, 49(6): 1049-1056.

7. Rippin JD, Patel A, Bain SC: Genetics of diabetic nephropathy. Best Pract Res Clin Endocrinol Metab 200 I, I 5(3):345-358.

8. Moczulski DK, Rogus JJ, Antonellis A, Warram JH, Krolewski AS: Major susceptibility locus for nephropathy in type I diabetes on chromosome 3q: results of novel discordant sib-pair analysis. Diabetes 1998, 47(7): I I64-II69.

9. Bowden DW, Sale M, Howard TD, Qadri A, Spray BJ, Rothschild CB, Akots G, Rich SS, Freedman BI: Linkage of genetic markers on human chromosomes 20 and 12 to NIDDM in Caucasian sib pairs with a history of diabetic nephropathy. Diabetes 1997, 46(5):882-886.

10. Imperatore G, Hanson RL, Pettitt DJ, Kobes S, Bennett PH, Knowler WC: Sib-pair linkage analysis for susceptibility genes for microvascular complications among Pima Indians with type 2 diabetes. Pima Diabetes Genes Group. Diabetes 1998 , 47(5):82I-830.

II. Vardarli I, Baier LI, Hanson RL, Akkoyun I, Fischer C, Rohmeiss P, Basci A, Bartram CR, Woude FJ Van Der, Janssen B: Gene for susceptibility to diabetic nephropathy in type 2 diabetes maps to 18q22.3-23. Kidney Int 2002, 62(6):2I76-2/83.

12. lyengar SK, Abboud HE, Goddard KA, Saad MF, Adler SG, Arar NH, Bowden DW, Duggirala R, Elston RC, Hanson RL, et al.: Genomewide scans for diabetic nephropathy and albuminuria in multiethnic populations: the family investigation of nephropathy and diabetes (FIND). Diabetes 2007, 56(6): I577-I585.

13. Ewens KG, George RA, Sharma K, Ziyadeh FN, Spielman RS: Assessment of 115 candidate genes for diabetic nephropathy by transmission/disequilibrium test. Diabetes 2005, 54(I I):3305-33 I8

14. Zhang W, Morris QD, Chang R, Shai O, Bakowski MA, Mitsakakis N, Mohammad N, Robinson MD, Zirngibl R, Somogyi E, et al.: The functional landscape of mouse gene expression. J Biol 2004, 3(5):2I.

15. Liang M, Cowley AW Jr, Hessner MJ, Lazar J, Basile DP, Pietrusz JL: Transcriptome analysis and kidney research: toward systems biology. Kidney Int 2005, 67(6):2 I I 4-2 I 22

16. Soutourina O, Cheval L, Doucet A: Global analysis of gene expression in mammalian kidney. Pflugers Arch 2005, 450(I): $13-25$

17. Susztak K, Sharma K, Schiffer M, McCue P, Ciccone E, Bottinger EP: Genomic strategies for diabetic nephropathy. J Am Soc Nephrol 2003, 14(8 Suppl 3):S27I-278.

18. Janssen U, Phillips AO, Floege J: Rodent models of nephropathy associated with type II diabetes. I Nephrol 1999, I 2(3): 159-172

19. Gauguier D, Froguel P, Parent V, Bernard C, Bihoreau MT, Portha B, James MR, Penicaud L, Lathrop M, Ktorza A: Chromosomal mapping of genetic loci associated with non-insulin dependent diabetes in the GK rat. Nat Genet 1996, I 2(I):38-43.

20. Phillips AO, Baboolal K, Riley S, Grone H, Janssen U, Steadman R, Williams J, Floege J: Association of prolonged hyperglycemia with glomerular hypertrophy and renal basement membrane thickening in the Goto Kakizaki model of non-insulindependent diabetes mellitus. Am J Kidney Dis 200I, 37(2):400-4I0.

21. Sato N, Komatsu K, Kurumatani H: Late onset of diabetic nephropathy in spontaneously diabetic GK rats. Am J Nephrol 2003. 23(5):334-342.

22. Gurley SB, Clare SE, Snow KP, Hu A, Meyer TW, Coffman TM: Impact of genetic background on nephropathy in diabetic mice. Am J Physiol Renal Physiol 2006, 290(I):F214-222.

23. Tesch GH, Allen T): Rodent models of streptozotocin-induced diabetic nephropathy. Nephrology 2007, I 2(3):26I-266.

24. Wilder SP, Kaisaki PJ, Argoud K, Ragoussis J, Bihoreau MT, Gauguier $\mathrm{D}$ : Comparative analysis of methods for gene transcription profiling data derived from different microarray technologies in rat and mouse models of diabetes. BMC Genomics 2009, 10:63.

25. Toye AA, Dumas ME, Blancher C, Rothwell AR, Fearnside JF, Wilder SP, Bihoreau MT, Cloarec O, Azzouzi I, Young S, et al.: Subtle metabolic and liver gene transcriptional changes underlie dietinduced fatty liver susceptibility in insulin-resistant mice. Diabetologia 2007, 50(9): | 867-1879. 
26. Schrijvers BF, De Vriese AS, Flyvbjerg A: From hyperglycemia to diabetic kidney disease: the role of metabolic, hemodynamic, intracellular factors and growth factors/cytokines. Endocr Rev 2004, 25(6):97I-1010.

27. Janssen B, Hohenadel D, Brinkkoetter P, Peters V, Rind N, Fischer C, Rychlik I, Cerna M, Romzova M, de Heer E, et al.: Carnosine as a protective factor in diabetic nephropathy: association with a leucine repeat of the carnosinase gene CNDP I. Diabetes 2005, 54(8):2320-2327.

28. Vionnet N, Tregouet D, Kazeem G, Gut I, Groop PH, Tarnow L, Parving $\mathrm{HH}$, Hadjadj S, Forsblom C, Farrall M, et al:: Analysis of 14 candidate genes for diabetic nephropathy on chromosome $3 \mathrm{q}$ in European populations: strongest evidence for association with a variant in the promoter region of the adiponectin gene. Diabetes 2006, 55(I I):3166-3I74.

29. Wilder SP, Bihoreau MT, Argoud K, Watanabe TK, Lathrop M, Gauguier D: Integration of the Rat Recombination and EST Maps in the Rat Genomic Sequence and Comparative Mapping Analysis With the Mouse Genome. Genome Res 2004, I 4(4):758-765.

30. Phillips AO, Janssen U, Floege J: Progression of diabetic nephropathy. Insights from cell culture studies and animal models. Kidney Blood Press Res 1999, 22(I-2):8I-97.

31. Susztak K, Bottinger E, Novetsky A, Liang D, Zhu Y, Ciccone E, Wu $D$, Dunn S, McCue P, Sharma K: Molecular profiling of diabetic mouse kidney reveals novel genes linked to glomerular disease. Diabetes 2004, 53(3):784-794.

32. Wallis RH, Collins SC, Kaisaki PJ, Argoud K, Wilder SP, Wallace KJ, Ria M, Ktorza A, Rorsman P, Bihoreau MT, et al.: Pathophysiological, genetic and gene expression features of a novel rodent model of the cardio-metabolic syndrome. PLOS ONE 2008, 3(8): 22962.

33. Alberts R, Terpstra P, Li Y, Breitling R, Nap JP, Jansen RC: Sequence polymorphisms cause many false cis eQTLs. PLOS ONE 2007, 2(7):e622.

34. Seubert JM, Xu F, Graves JP, Collins JB, Sieber SO, Paules RS, Kroetz $\mathrm{DL}$, Zeldin DC: Differential renal gene expression in prehypertensive and hypertensive spontaneously hypertensive rats. Am J Physiol Renal Physiol 2005, 289(3):F552-56I.

35. Nobrega MA, Fleming S, Roman RJ, Shiozawa M, Schlick N, Lazar J, Jacob HJ: Initial characterization of a rat model of diabetic nephropathy. Diabetes 2004, 53(3):735-742.

36. Cheng Z], Vaskonen T, Tikkanen I, Nurminen $\mathrm{K}$, Ruskoaho $\mathrm{H}$ Vapaatalo H, Muller D, Park JK, Luft FC, Mervaala EM: Endothelial dysfunction and salt-sensitive hypertension in spontaneously diabetic Goto-Kakizaki rats. Hypertension 200I, 37(2 Part 2):433-439.

37. Rees DA, Alcolado JC: Animal models of diabetes mellitus. Diabet Med 2005, 22(4):359-370.

38. Yip J, Mattock MB, Morocutti A, Sethi M, Trevisan R, Viberti G: Insulin resistance in insulin-dependent diabetic patients with microalbuminuria. Lancet 1993, 342(8876):883-887.

39. Knoll KE, Pietrusz JL, Liang M: Tissue-specific transcriptome responses in rats with early streptozotocin-induced diabetes. Physiol Genomics 2005, 2 I (2):222-229.

40. Wada J, Zhang H, Tsuchiyama Y, Hiragushi K, Hida K, Shikata K, Kanwar YS, Makino H: Gene expression profile in streptozotocininduced diabetic mice kidneys undergoing glomerulosclerosis. Kidney Int 200I, 59(4): I363-I373.

4I. Martini S, Eichinger F, Nair V, Kretzler M: Defining human diabetic nephropathy on the molecular level: integration of transcriptomic profiles with biological knowledge. Rev Endocr Metab Disord 2008, 9(4):267-274.

42. Baelde HJ, Eikmans M, Doran PP, Lappin DW, de Heer E, Bruijn JA Gene expression profiling in glomeruli from human kidneys with diabetic nephropathy. Am J Kidney Dis 2004, 43(4):636-650.

43. Lappin DW, McMahon R, Murphy M, Brady HR: Gremlin: an example of the re-emergence of developmental programmes in diabetic nephropathy. Nephrol Dial Transplant 2002, I 7(Suppl 9):65-67.

44. Taupin D, Podolsky DK: Trefoil factors: initiators of mucosal healing. Nat Rev Mol Cell Biol 2003, 4(9):72I-732.

45. Klahr S: The bone morphogenetic proteins (BMPs). Their role in renal fibrosis and renal function. J Nephrol 2003, I6(2): 179-185.
46. Gupta S, Clarkson MR, Duggan J, Brady HR: Connective tissue growth factor: potential role in glomerulosclerosis and tubulointerstitial fibrosis. Kidney Int 2000, 58(4): I389- 1399.

47. Hayes JD, Flanagan JU, Jowsey IR: Glutathione transferases. Annu Rev Pharmacol Toxicol 2005, 45:5I-88

48. Smith CP, Fenton RA: Genomic organization of the mammalian SLCI4a2 urea transporter genes. J Membr Biol 2006, 212(2): 109-117.

49. Kausalya PJ, Amasheh S, Gunzel D, Wurps H, Muller D, Fromm M, Hunziker W: Disease-associated mutations affect intracellular traffic and paracellular $\mathbf{M g 2 +}$ transport function of Claudin16. I Clin Invest 2006, I I6(4):878-89|

50. Hou J, Shan Q, Wang T, Gomes AS, Yan Q, Paul DL, Bleich M, Goodenough DA: Transgenic RNAi depletion of claudin- 16 and the renal handling of magnesium. I Biol Chem 2007, 282(23): $|7||4-| 7 \mid 22$.

5I. Sadlier DM, Connolly SB, Kieran NE, Roxburgh S, Brazil DP, Kairaitis L, Wang Y, Harris DC, Doran P, Brady HR: Sequential extracellular matrix-focused and baited-global cluster analysis of serial transcriptomic profiles identifies candidate modulators of renal tubulointerstitial fibrosis in murine adriamycininduced nephropathy. J Biol Chem 2004, 279(28):29670-29680.

52. Halama N, Yard-Breedijk A, Vardarli I, Akkoyun I, Yard B, Janssen B, Woude FJ van der: The Kruppel-like zinc-finger gene ZNF236 is alternatively spliced and excluded as susceptibility gene for diabetic nephropathy. Genomics 2003, 82(3):406-4II.

\section{Pre-publication history}

The pre-publication history for this paper can be accessed here:

http://www.biomedcentral.com/1755-8794/2/41/prepub
Publish with Biomed Central and every scientist can read your work free of charge

"BioMed Central will be the most significant development for disseminating the results of biomedical research in our lifetime. "

Sir Paul Nurse, Cancer Research UK

Your research papers will be:

- available free of charge to the entire biomedical community

- peer reviewed and published immediately upon acceptance

- cited in PubMed and archived on PubMed Central

- yours - you keep the copyright
BiolMedcentral 